\title{
An industrial case study of immediate benefits of requirements engineering process improvement at the Australian Center for Unisys Software
}

\author{
Daniela Damian \\ Dept. of Computer Science \\ University of Victoria, BC \\ PO Box 3055, BC \\ Canada, V8W 3P6 \\ DanielaD@uvic.ca
}

\author{
Didar Zowghi \\ Faculty of Information Technology \\ University of Technology, Sydney \\ PO Box 123, Broadway \\ NSW 2007, Australia \\ didar@it.uts.edu.au
}

\author{
Lakshminarayanan \\ Vaidyanathasamy and Yogendra \\ Pal \\ Unisys Australia Limited \\ 1C Homebush Bay Drive \\ Rhodes, NSW 2138, Australia \\ \{Lnv.Samy, \\ Yogendra.Pal\}@unisys.com
}

\begin{abstract}
This paper describes an industrial experience in process improvement at one of the Unisys development labs in Australia. Following a Capability Maturity Model (CMM) mini-assessment, the organization is undertaking significant changes in the requirements management process, which include the introduction of group session approaches to requirements analysis and a structured method for writing requirements. An empirical evaluation which investigated other aspects of the process improvement than the CMM model indicates tangible benefits as well as perceived long-term benefits during design and testing. Findings confirm that a more thorough requirements analysis results in more clearly defined, better understood and specified requirements, and an enhanced ability to address the market needs and product strategy requirements. The catalyst behind these improvements included project management leadership, managing the human dimension, collaboration among stakeholders and senior management support.
\end{abstract}

Keywords: requirements engineering, process improvement, industrial case study, empirical assessment 


\section{Introduction}

Efforts to improve software process have been going on for several decades. Software engineering has witnessed the development of models and standards for software process assessment and improvement such as the ISO 9001 standard for quality management systems (Quality Standards, 1987), and the Software Engineering Institute's Capability Maturity Model for Software (CMM) (CMM, 1991; Paulk et al., 1993; SEI, 1995). During the 1990s published reports of successful process improvement programs began to emerge from both single and multisite organizations such as Hughes (Humphrey et al., 1991), Raytheon (Dion, 1993), and Motorola (Diaz and Sligo, 1997).

Requirements management, however, does not benefit from such a set of standard and well-accepted guidelines on "best practice" or from sound Requirements Engineering (RE) process improvement assessments. Although a relatively new field in software engineering, Requirements Engineering has received increased interest from both academics and practitioners as one of the more important, but difficult and problematic phases of system development (Chaos Report, 1995). What has emerged within the RE research community in recent years, however, indicates that without knowing enough about the organization, systems engineering, software development processes and the type of software being developed, it is difficult to prescribe a "best practice" for RE. While Sommerville et al $(1997 ; 1998)$ have developed a comprehensive set of guidelines for good practices in RE, the empirical evidence about actual improvements due to improved RE practice offers little comfort to the practitioner. These same guides warn that revolutionary change is not practical, that instead careful evolutionary improvements are more fruitful. Practitioners are encouraged to measure results to gauge effectiveness, but topics such as empirical evidence are largely left to the imagination, as an exercise for the reader. Further, studies that investigate the staff perception of the introduction of well-defined RE processes in deve lopment contexts where little or no prior attention was paid to requirements management are truly lacking in the literature.

In this paper we describe an empirical assessment of RE process improvement at one of the Unisys development laboratories, the Australian Center for Unisys Software (ACUS). Our report addresses the improvement in requirements management, one of the significant challenges faced by the organization. While the organization was undertaking a larger CMM process improvement initiative, our study focused on understanding the practice and effects of improved requirements management at ACUS, in addition to the evidence available from the CMM assessment. Given the scarcity of practical guidelines on "good RE”, this paper intends to provide practitioners with insights into immediate (i.e. early in the software process) benefits that improved RE practice bring to an organization such as ACUS. While CMM assessments are valuable resources in informing that an organization is following a well-defined process, our investigation sought to discover, besides perceived immediate benefits in the software development, the relationship between improved RE processes and improved developer communication, project scope 
management and requirements negotiations, developer participation in requirements definition, and staff morale in response to cultural change.

To the research community, this study is informing about a particular use of specific RE methods in a programme of process improvement, and provides evidence that could potentially lead to the development of a theory of RE improvement. To software practitioners, this study provides an indication that RE process improvements can provide clear benefits to the organization; an account of the effects of improved RE practice at ACUS not only adds to our understanding of good requirements practice but also provides strong motivation for software organizations to develop programs for improvement of their own requirements processes.

The paper begins in Section 2 with a discussion of related work in software process improvement and in particular requirements engineering improvement areas. While our research method is described in Section 3, background information on the organization and its challenges in aligning customer (market) needs with strategy directions are summarized in Section 4. Section 5 presents the initiative for requirements management process improvement undertaken by ACUS, being followed by a detailed description of our empirical assessment and its findings in Sections 6 and 7 respectively. Limitations of our research are discussed in Section 8 and an outline of our future research concludes the paper in Section 9.

\section{Related work}

Software Process Improvement (SPI) has become an increasingly popular approach for achieving improvements in the quality of software processes and products. SI provides organizations with a powerful means of assessing their current capabilities for developing software systems and in doing so, identifying their weaknesses and strengths. SPI models such as the Capability Maturity Model (CMM)® (Paulk et al., 1993; SEI, 1995), and standards such as ISO's SPICE or the emerging ISO/IEC 15504 international standard (El Emam et al., 1998; ISO, 1998) specifically prescribe processes for achieving high quality software.

It is reported that SPI generally delivers substantial benefits (Humphrey et al., 1991; Johnson, 1994). Research results show that companies with high level of maturity benefit from increased product quality, improved customer satisfaction and reduced risk (Herbsleb and Goldenson, 1996). The SPI literature contains many case studies of successful companies and the description of their SPI programs. For example El Emam and Briand (1997) reviewed and summarized the empirical evidence on the costs and benefits of SPI up to 1997. In this review, they report that the assessment of SPI effort led by the Schlumberger Laboratory for Computer Science have reported several important benefits (Wohlwend and Rosenbaum, 1993). These include: improved project communication, customer reports confirmed improved product quality, improved time to market by reducing requirement validation cycles, defect density in product was halved, and one group more than doubled its productivity. Brodman and Johnson 
(1996) collected SPI data from 33 companies using questionnaires and interviews and reported that some organizations witnessed increased productivity, reduced defects and rework effort, while others benefited from less overtime and improved cooperation between functional groups. Success factors of the organizational change in software process improvements include: management commitment and support, staff involvement, providing enhanced understanding, tailoring improvement initiative, managing the improvement project and others (Stelzer and Mellis, 1998). Lessons learned from long term studies such as work by Basili et al (2002), which examined 25 years of SPI effort in NASA, draw attention to the critical nature of some of these factors such as management buy-in to the process. Majority of these studies, however, have looked at the benefits of SPI without any reference to what the perception of the staff may have been before and after the SPI initiatives began, neither do they examine the phenomenon at early stages of implementation of SPI initiative. In this study we intended to examine the Requirements Engineering process improvement at early stages in the development process, and in particular to understand the software engineers' reaction to revised requirements processes, and signs of organizational culture change with respect to how requirements were analyzed and managed during the development.

The work of Hall et al (2002) provides empirical evidence on the requirements related problems that twelve software companies in UK faced. Their results suggest that patterns of requirements problems are related to the level of companies' maturity. In other words, the higher the CMM maturity level of the company the less frequent are the requirements problems. Unfortunately, the role that requirements engineering practices play in SPI has not been addressed by previous work.

The typical approach to CMM-based SPI in the software engineering community ${ }^{1}$ is to identify Key Process Areas (KPA). SPI models broadly recommend best practice based on a specific sequence of implementation, hence providing a predefined path for improvement. Some of these models include the requirements engineering process within their scope and define what are believed to be best requirements engineering practices. While CMM has a KPA on requirements management (SEI, 1995), the ISO/IEC 15504 international standard defines a "develop software requirements" process (ISO, 1998). More recently the importance of RE process has attracted attention as a separate process in its own right that demands to be separately investigated and assessed. To the best of our knowledge, the only maturity model specific to RE process has been defined by Sommerville and Sawyer including a catalogue of RE good practices organized in a recommended order of implementation (Sommerville and Sawyer, 1997; Sawyer et al., 1998).

Process improvement in these models almost always involves repeated assessment of the process. This type of assessment generally provides a quantitative score reflecting the extent of an organization's implementation of best practices as defined in the particular assessment model being used. The extent to

\footnotetext{
${ }^{1}$ See for example the proceedings of the Software Engineering Process Group conferences 96-99
} 
which these best practices are adopted by organizations determines how high the score will be. The score obtained as a result of these assessments then becomes the basis for making future decisions on investing in process improvement activities, and also allows for tracking the improvement efforts in the organizations. The basic assumption of these approaches to SPI is that improving RE practices using a specific assessment model is expected to result in better project or organizational performance. This is termed the predictive validity of the process capability score (El Emam and Birk, 2000).

Empirical assessments of SPI reported in the research literature so far have failed to produce strong evidence that there is indeed a relationship between assessment score and project or organizational performance. However, important to note is the empirical study by El Emam and Birk (2000) which investigated the relationship between the capability of the software requirements analysis (SRA) process as defined in ISO/IEC 15504 international standard and the performance of software projects. The study was conducted in the context of the SPICE Trials. This may be considered as one of the first studies to evaluate the predictive validity of SRA process capability using an internationally standardized measure of process capability. Their results indicate that for large organizations, SRA process capability (as measured in ISO/IEC 15504) is related to project productivity. This means that improvements in SRA process capability are associated with a reduction in the cost of software projects (in terms of the amount of rework during the project). However, no relationship was found with other measures of performance, nor was there any relationship between SRA process capability and any of the performance measures that were used for small organizations. In this case study of a relatively medium organization (around 100 software engineers) we researched immediate benefits of improved RE practice (such as better problem definition and communication during design) as a means of studying the relationship between the capability of improved RE practice and project performance. Having conducted the study early in the development process, performance measures such as productivity were not possible; instead, perceived benefits as expressed by 'expert designers' were more appropriate and valuable.

\section{Research method}

This research was conducted as an industrial case study at ACUS over a period of eight months (Aug 2001 to April 2002). Data collection methods included the administration of two questionnaires, inspection of requirements documents, observations of requirements analysis and negotiation sessions, as well as interviews with ACUS engineering and management staff. To our best knowledge, the scarce research in empirical studies of requirements engineering process improvement does not provide welldefined instruments such as questionnaire items or interview questions for such assessments. The questionnaires and interview questions were developed therefore to inquire about the specific revisions of the requirements engineering process. As outlined in the Appendix, the questionnaire items solicited input about the effectiveness of the revised RE process (i.e. Q1 in Appendix), and in particular of the specific 
process components (Q2 through Q4), and the extent to which they brought an improvement (Q6). While question 5 addressed the role of training in the implementation of the revised process, questions 7 though 9 referred to potential immediate and long-term benefits of the revised RE process in software development practices at ACUS.

Thirty-four members of the software engineering, management and product information were involved in questionnaires, interviews, and requirements analysis and negotiation sessions. Given that historical project data was limited, we relied on the extensive professional experience of engineers at ACUS to provide comparison to previous practice. Thus the criteria on which the study participants were selected included participation in previous projects at ACUS (which used the RE process before the improvement initiative) and naturally involvement in the current projects. The interviews conducted mainly with senior management and team leaders were semi-structured and provided an opportunity to explore the ways in which the revised RE process was different from previous practice and the potential impact on the software development practice at ACUS. Follow-up interviews were conducted often after the analysis of the questionnaire data, or after the observation of certain requirements negotiation sessions. By interviewing senior project members from different functional departments (e.g. engineering, documentation and management), an attempt was made to collect information from multiple viewpoints and thus to obtain a comprehensive view of the RE process improvement at ACUS. Respondents were encouraged to be critical of their experiences with the new processes in an effort to further improve them. Therefore we believe that this evidence can be regarded as an objective assessment rather than the hopeful expectations of participants of the process.

The majority of our evidence was collected anonymously to protect respondents from the scrutiny of the organization. In particular, the names of participants involved were largely unknown to ACUS senior management, to remove the possibility of apprehension to provide accurate information. To further avoid the risk of a perceived apprehension towards job loss if confidential data is provided to the senior management, the questionnaires were administered in paper copies and collected individually by the first author. Similarly, the first author sat through all requirements analysis sessions and negotiation meetings as defined by the revised process. Extensive motes and observation logs of the discussions relevant to requirements were kept, in an attempt to identify issues that informed the analysis of the other data in the study.

\section{Background information: the organization and its RE practice}

ACUS is an ISO 9001 certified software development organization, developing product-line software, that belongs to an international multi-site organization with headquarters and marketing divisions in US. Features for new releases are a result of the agreement between the US business unit and ACUS which considers both market needs (representing current as well as potential customer needs) and product 
strategy requirements (representing technology and engineering direction of the product in line with the organizational strategy).

When this study began, ACUS suffered from a lack of experience with a formal requirements management process in a setting where major stakeholders are geographically distributed across several continents. The Business Initiative (BI) Group in US compiled requirements in a document containing one-line statements of intent, and communicated the document to the Engineering group at ACUS in Australia for implementation. Similarly, major customer groups are scattered world-wide and direct communication with ACUS was minimal. After the product enhancement features were communicated to ACUS, ongoing negotiations between BI and Engineering were necessary in managing the scope of a release. While the Engineering input was very important in providing development estimates and feasibility assessments, BI Group was a major player in decision making.

\section{1 The Challenge}

This requirements management process required improvement. The specific challenges faced by the organization in meeting market needs and product strategy requirements included the following:

- Requirements were not clearly defined or documented. They were communicated as high level 'system features' often ambiguous in stating the required functionality and difficult to determine the nuances of possible future changes.

- Requirements were not fully understood by developers. Most often the rationale for the requirements was missing or buried in a database of customer requests, making it difficult to obtain full understanding of the customers' intended or actual usage of the system

- Negotiations between ACUS and BI were ineffective because ACUS Engineering management had difficulty in (1) understanding the market/business direction clearly and aligning it with the engineering/technology strategy and (2) providing estimates of development effort, since the high level 'system features' did not provide sufficient information

- Inadequate change management practice. Traceability links to the rationale of the requirement, or to other requirements were not adequately maintained, often leading to inadequate change impact analysis and consequently poor project scope management.

As a result, requirements creep was prevalent throughout the development life cycle. A larger number of features were initially committed to, compared to what was delivered, resulting in the perception that ACUS was delivering less than optimal quality software. Initial over commitment by ACUS combined with a poor change impact analysis led to schedule overruns and dropped functionality. This, in turn, resulted in the perception that ACUS delivered sub-optimal quality software. 
In September 2001 a CMM mini assessment of the software process was conducted. The objective was to identify priorities for improvement to achieve Level 2 by July 2002. Requirements management was identified as one Key Process Area for improvement, together with other areas such as SQA, Project Planning and Project Tracking. The diagram in Figure 1 places the Requirements Management process improvement in the context of the overall process improvement initiative at ACUS. The main challenge of meeting market needs and product strategy requirements is shown together with the specific challenges in the area of Requirements Management (outlined in the lower-left corner of the diagram). In this paper we report on the efforts to improve requirements management practices, the other areas being only briefly included in the diagram for completeness.

Efforts for improvement started soon after assessment. A focused initiative was introduced to improve practice with a view to learn from mistakes made on previous projects and using the CMM as the framework. The process improvement initiative had appropriate infrastructure: a software process improvement steering committee and a Software Engineering Process Group (SEPG) was established, the process improvement effort had middle and senior management support, and an action plan was developed. The focus of this paper describes the efforts to improveme RE practice and the subsequent empirical assessment of this improvement when the "requirements phase" completed. The next section outlines the strategy for improvement in requirements practice and its implementation plan at ACUS.

\section{Process improvement initiative}

The strategy for improvement was to tailor the standard organization-wide (Unisys) RE practice to meet the specific needs at ACUS. In particular, the strategy had five steps:

1. Define high level polic y and reach agreement with management and stakeholders

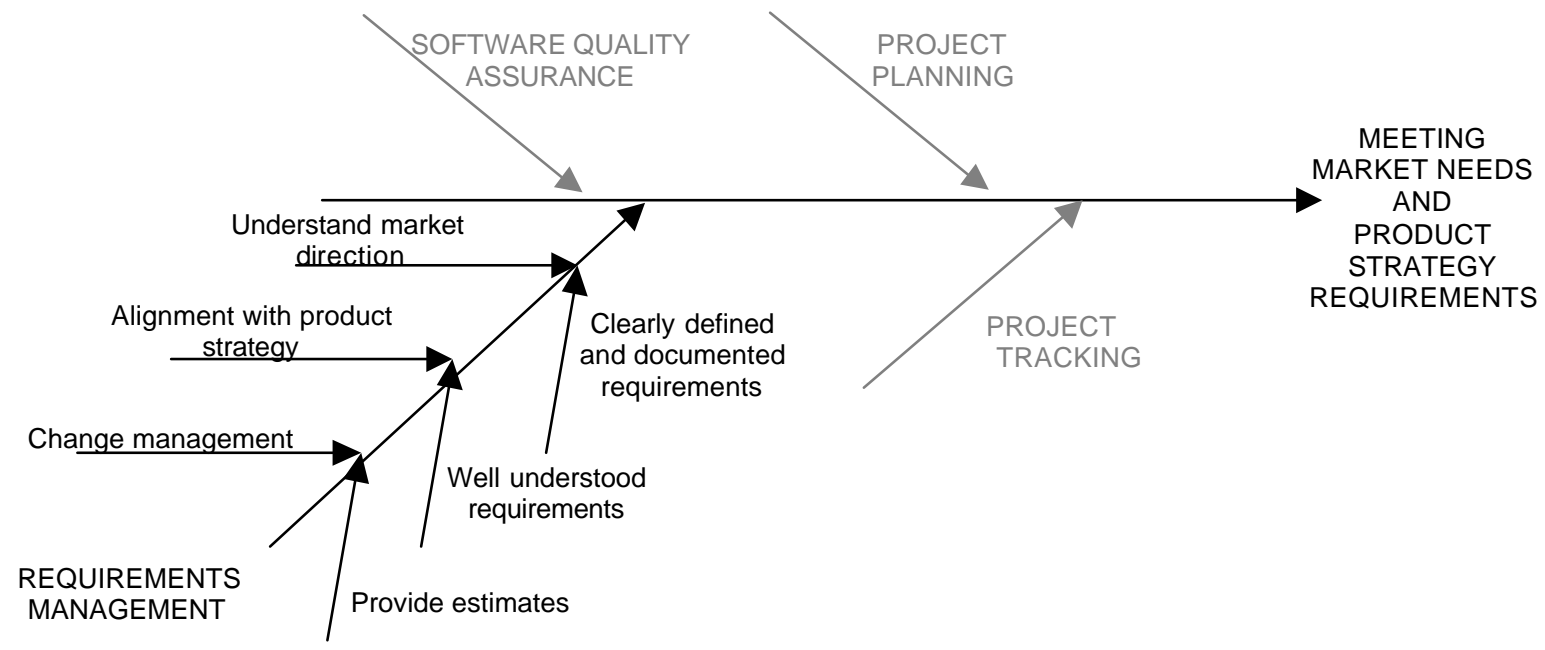

Figure 1. A cause-effect diagram to illustrate the major challenge (meeting market needs and product strategy requirements) and the specific challenges in the requirements management practice 
2. Revise and refine the RE process to address the current problems;

3. Support the process with methods and appropriate tools and

4. Provide training and leadership in the utilization of the revised process, and associated methods and tools.

5. On going reviews and assessments for continuous improvement

\subsection{Implementation plan}

Before we describe the revised RE process and its supporting methods and tools, we illustrate the elements of this strategy in the context of the development life cycle. As illustrated in Figure 2, immediately after the miniassessment in September 2001, training on the revised process as well as the supporting methods and tools started. The objective of the improvement efforts was to achieve better feature analysis, with the ambitious goal of achieving a better understanding and specification of features as communicated by the Business Initiative group. This effort was coordinated by the Project Manager at ACUS, who revised the process, initiated training, and led the requirements analysis sessions. The outcomes of these sessions were recorded in a Requirements Specification document, reviewed internally at ACUS and then distributed for validation with the BI group in US and representative customers. The intention was (1) provide developers with a better understanding of system features and associated software requirements in their daily work as well as (2) to aid decision making during requirements negotiations. In the requirements negotiations between the BI and ACUS management, enhanced understanding of requirements would assist not only engineering in providing estimates on development effort; but also to assist the decision makers in the prioritization of requirements and managing requirements scope. Furthermore, there was the expectation that the improved RE practice would have positive impact during subsequent phases such as design, coding and testing.

In Figure 2, our investigation is illustrated in the form of two questionnaires: the first one immediately after training was conducted and the requirements analysis sessions were initiated, to assess initial feedback with the new RE practice at ACUS; the second one was more detailed and collected information on perceived benefits of he new practice, after participants' active involvement in the requirements analysis stage.

\subsection{Process improvement critical success factors}

For the assessment to be meaningful, critical success factors were defined in relation to the six major challenges faced by the organization in requirements management, as identified in the diagram illustrated in Figure 1. During software development, the process was considered to have improved if:

1. Requirements were clearly defined and documented,

2. Features and the associated software requirements were well understood,

3. There was a clear understanding of the alignment between market direction and product strategy requirements 
4. The software engineers and mangers were able to provide better estimates on development effort, and

5. There was more effective tracking of requirements changes and their affected development artifacts (such as design documents and test plans) through appropriate requirements change management techniques.

Assessing the improvement in relation to these factors requires the collection of data throughout the development life cycle. For some of these factors, early assessment is meaningful; for others, assessment is needed after the software is released to customers. We believe it is possible to observe whether better requirements understanding, clearly defined, documented requirements and enhanced ability to provide development estimates had been achieved early in the development cycle, possibly right after requirements analysis. In contrast, to determine the benefits of better requirement change management or improved understanding of market and strategic directions could only be fully assessed towards the end of the development life-cycle, when the product is tested and released to customers.

Besides attempting to address all factors above, our study also collected data on perceived immediate and long-term benefits of the revised requirements management practice, together with other issues such as training. Further empirical assessment is needed to reconfirm some of these perceived benefits, as well as to complete the assessment as related to the fifth critical success factor at the end of the development life-cycle.

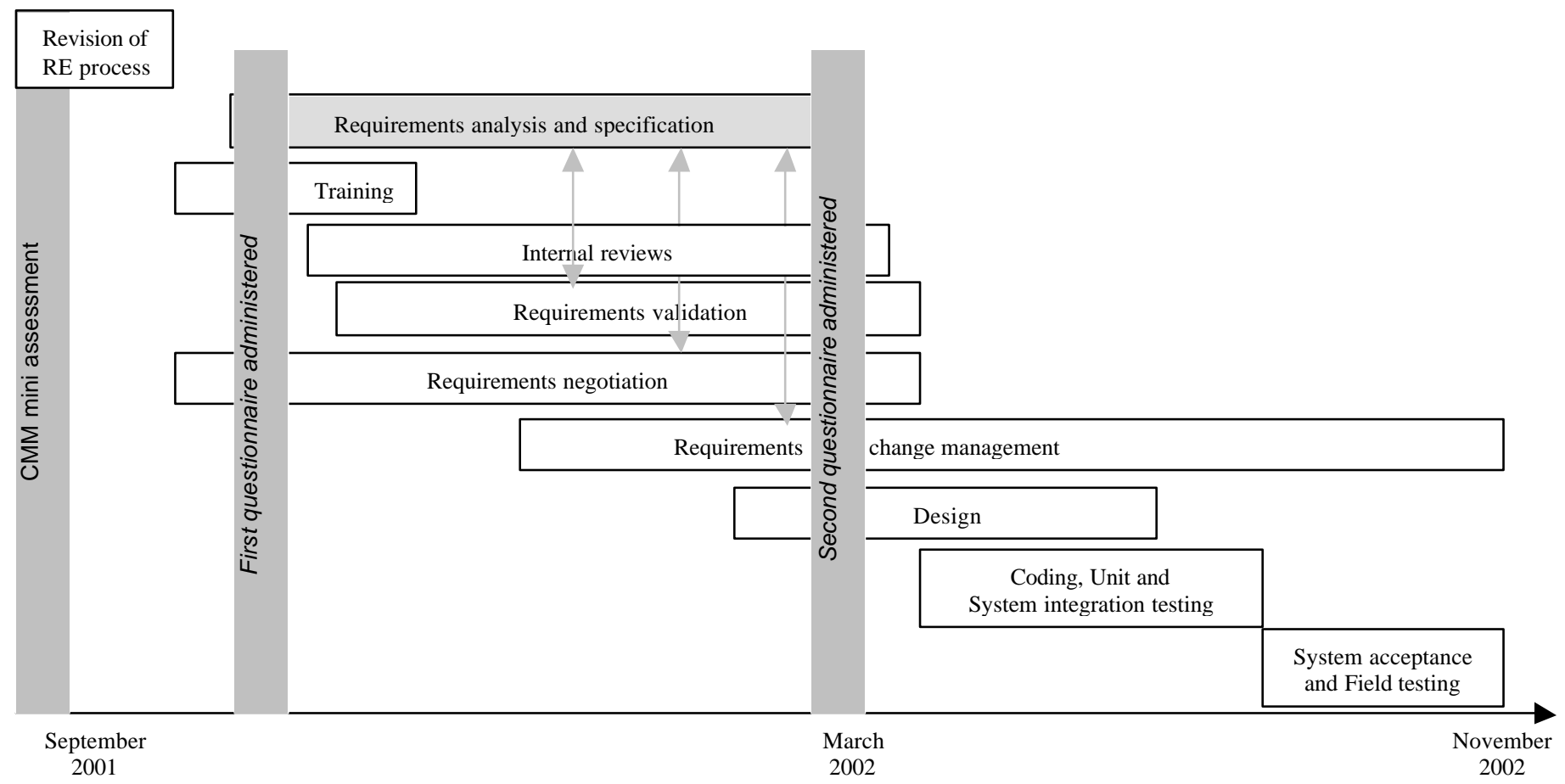

Figure 2. Implementation plan 


\subsection{Changes implemented}

In this section we describe the revised RE process together with the methods and tools employed to support it.

\subsubsection{A revised RE process}

The RE process was tailored to address current challenges as follows:

1. Provide better analysis and description of the required 'system features' by:

- decomposing the high level 'system features' into software requirements,

- defining traceability links between features and associated software requirements, requirements and rationale, and requirements to test method and test scenario

2. Conduct better validation of requirements with BI group in US and customers world-wide

3. Improve requirements tracking through the development life-cycle by using a requirements management tool

\subsubsection{Supporting methods and tools}

This process was supported by the following methods and tools:

- Group analysis sessions to achieve a better understanding of each feature. The sessions involve participants with relevant knowledge and expertise in the particular feature, from several functional areas: development, testing and product information. Each feature was analyzed within the existing product architecture; for each relationship identified within the architecture, more specific software requirements were

Full text requirement: When in full operation the computer system shall provide thirty per cent reserve in channel capacity.

Initiator of Action: The computer system Conditions for Action: in full operation Action: shall provide

Constraints for Action:

Object of Action: reserve in channel

Refinement/Source of capacity Object of $30 \%$

Refinement/Destination of Action:

Box 1. Sentence template constructs (in bold) and an example of a full text requirement parsed with the sentence template (used with the permission of Halligan, R. (11)) identified. The supporting tool was a mind map (see Figure 3) created for each feature - referred to "context diagram", to illustrate the impact of that required functionality in the system.

- Each software requirement was described using a sentence template (Halligan, 2000), with defined constructs outlined in Box 1. As seen in the foreground of Figure 3, an Excel Form is used to capture the elements of the sentence template and then saved in an Excel spreadsheet (see Figure 4). Hence these sessions were referred to as "requirements capture" sessions.

- For each software requirement, the rationale as well as a test method and test scenario were also captured in the Excel Form.

- After the completion of each requirements capture session, the information from the Excel file was stored in the Requirements Specification document and managed using Rational's RequisitePro ${ }^{\mathrm{TM}}$ requirements management tool. 


\subsubsection{Training: managing the human dimension}

Once the RE process was revised, training and leadership was essential to guide ACUS through the organizational change required to implement the new process. This aspect is often overlooked and becomes a cause of failure for organizational change efforts. One of the major difficulty in process improvement is the human dimension (Laporte and Trudel, 1998) and the importance of educating the software engineers in the methods used in the new practice emerged.

This organization had to contend with issues that most other organizations experience, including: breaking down the resistance to the introduction of formal requirements engineering, providing education on the downstream benefits of RE; harnessing the desire to improve processes; and introducing tools to support the revised RE process. This effort was actively led by the Project Manager, who had prior experience in proven RE methods and the resolve to manage the change. He designed workshops on the revised RE process and supporting methods and tools Furthermore, he actively facilitated around two thirds of the requirements capturing sessions.

Although a large number of developers attended training to address the issues above, more developers became active in the process, without formal training. Hence a hands-on approach to learning about the process and the methods was used for these developers.

\section{Findings}

In brief, the assessment of the RE process improvement at ACUS identified an initial enthusiasm with the revised process and methods; enthusiasm that was sustained throughout the observed period of aght months. Major efforts were made to improve the requirements analysis stage, which in turn enabled better requirements validation and negotiation with the headquarters in US. The findings suggest that more thorough analysis of the high-level system features resulted in clearer, better understood requirements, and

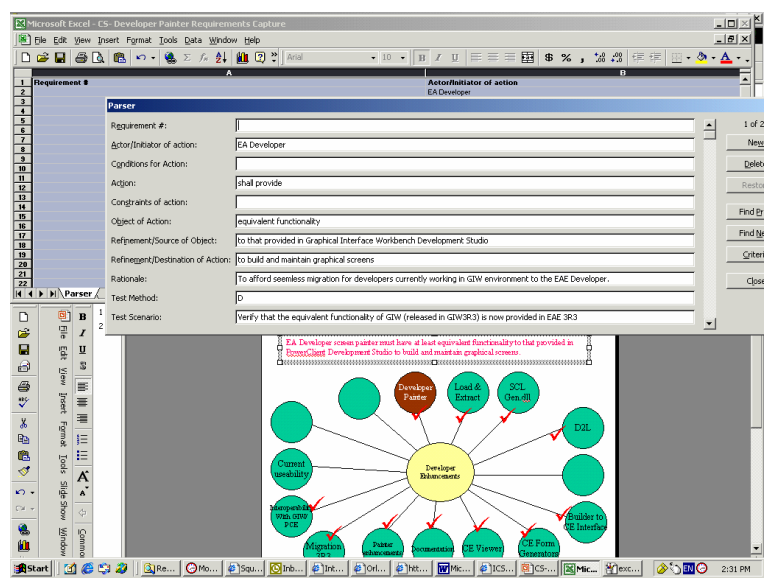

Figure 3. The "context diagram" used in the analy sis of each feature, and the Excel Form capturing the constructs of the sentence template

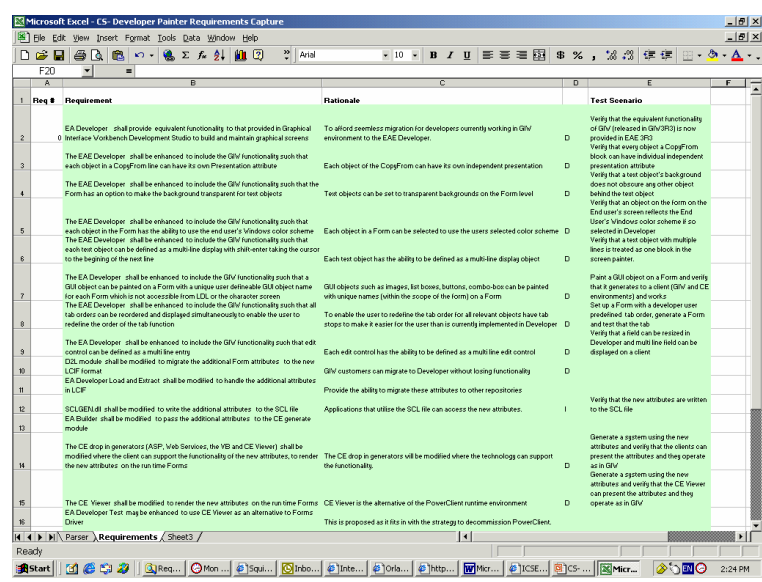

Figure 4. The requirements "captured" in an Excel file before being translated in the RS (small size to preserve confidentiality) 
an enhanced ability to address market needs and product strategy requirements. A tangible secondary benefit was the resulting close co-operation between different cross-functional groups involved in the development of the product. The findings reveal a significant association between the level of involvement in requirements analysis sessions and perceived effectiveness of improved RE practice at ACUS. Active involvement of staff across development platforms and from several functional areas (i.e. engineering, testing, product information) was found as one of the strengths in the improved practice, since it enabled an understanding of features within the entire system. A similar association was found in relation to a long-term perceived benefit, in that the

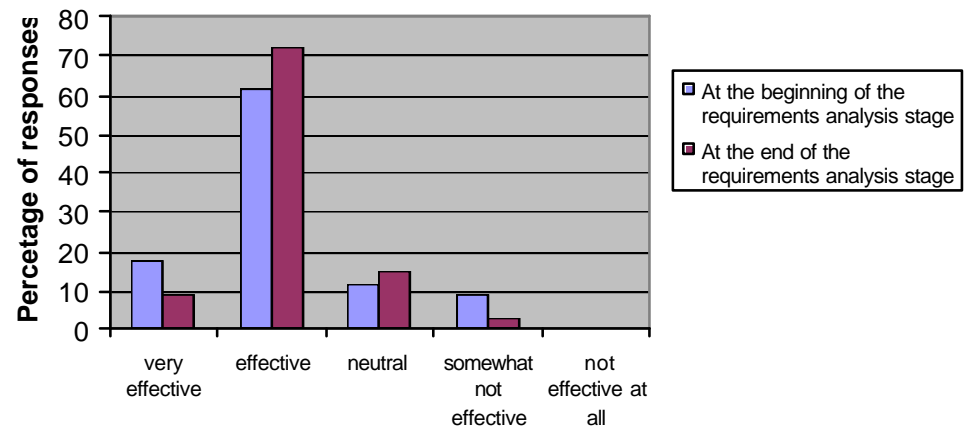
participants believed that effective requirements analysis has a direct

Figure 5. Effectiveness of "requirements capture" meetings at the beginning and end of the analysis stage

relationship to improved design activities.

The empirical assessment and these findings are described in detail in the sections that follow.

\subsection{Positive feedback from the start of the requirements analysis}

A first questionnaire was administered to thirty-four participants who had attended an average of 3 requirements capture sessions. Of primary interest was feedback from the participants with respect to the effectiveness of the newly introduced "requirements capture" sessions, as compared to previous practice at ACUS (see questionnaire in Appendix); the objective of these sessions was to analyze and understand high-level system features in the current context of the system, derive software requirements and increase shared understanding of required functionality.

As shown in Figure 5, there was a positive reaction to these sessions at the beginning of the analysis stage: $80 \%$ of participants reported effective to very effective meetings $(62 \%$ : effective, while 18\%: very effective). The graph aso shows the responses on the effectiveness of these meetings at the end of the requirements analysis stage (33 participants), which will be discussed later.

The requirements capture meetings employed (1) "context diagrams" for the analysis of system features in the context of the existing system architecture and (2) a sentence template for writing requirements as described in Box 1. Given that this method was completely new to the software engineers, we collected initial feedback on the effectiveness of this method in analyzing and writing requirements. The goal was to assess (1) positive aspects of the methods to determine the extent they contributed to:

(a) reducing the ambiguity of requirements,

(b) reducing time spent in capturing requirements 
(c) writing correct requirements, (d) complete requirements, and (e) consistent requirements,

(f) increased understanding of requirements

(g) discovering new (or unstated) requirements

(h) improving the structure of requirements

(i) discovering the relationships between the individual requirements as well as (2) negative aspects:

(j) inhibiting or

(k) (k) constraining the participants' thinking about or expressing the requirements.

Data was colleted by means of questions 2-4 in the questionnaire (see Appendix). Figure 6 illustrates the responses from 24 participants, on each of these questionnaire items (only the responses from participants involved in both questionnaires and more than 3 requirements capture sessions were considered). As indicated, 50-65\% of participants responded favorably to nearly all the items, with only a few exceptions: the new method did not appear, at the beginning, to reduce the time spent in capturing requirements (see (d) in Figure 6), to help discover unstated requirements ((g) in Figure 6) nor discover relationships between individual requirements (i). However, the overall response represent feedback which is fairly positive given that the new method had just been introduced in the RE process. The initial reaction to the use of the context diagram was also very positive (21 out of 24 respondents reported that the context diagram was helpful in analyzing the requirements). This evidence can be attributed to an initial enthusiasm with a more thorough analysis of requirements in the organization and we decided to include questions 2 and 4 (see Appendix) in the follow-up questionnaire at the end of the requirements analysis stage. The results are also shown in Figure 6 and are discussed later.

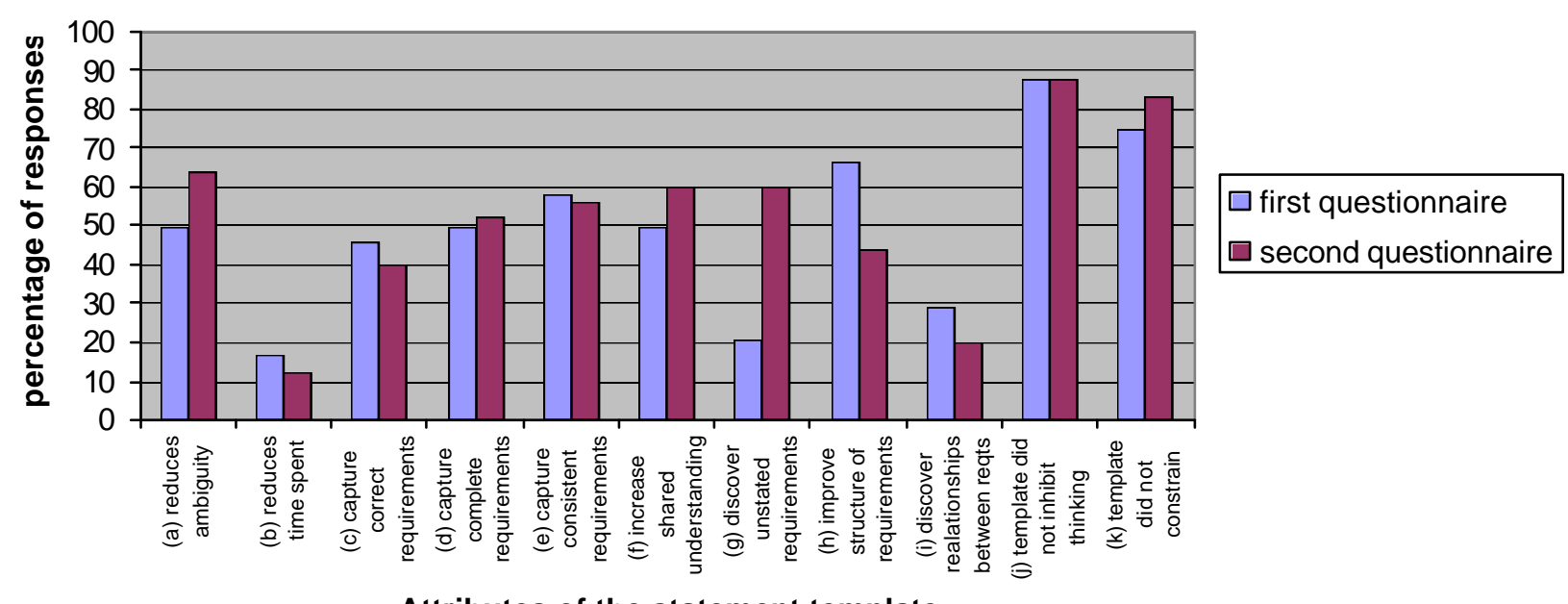

Attributes of the statement template

Figure 6. Feedback on the effectiveness of the requirements analysis and writing method, data from the first and second questionnaires 
An analysis of the qualitative data provides an explanation for this enthusiasm. Asked to comment on possible improvements from the previous practice in RE activities at ACUS, the participants unanimously mentioned specific benefits of the revised process. They noted a particular strength of the requirements analysis sessions was the group session approach which involved relevant software engineers, testing engineers and product information specialists in the analysis of features, i.e. "[improvement] by bringing a wider audience in at the start to uncover issues they can reduce the incidence of nasty surprises later on; the multi-disciplinary, multi-skilled team approach fosters concurrent engineering and shared vision in design." 2 These sessions subsequently enabled sharing the understanding of required functionality not only among developers, but also among those who need to test and document the functionality later in the development.

The previous practice at ACUS was weak in the area of systematic analysis or documentation of the requirements; the marketing and business requirements were communicated as one-line statements, leaving the software engineers not fully understanding the rationale behind the requirement, or the relationship with market needs or business strategy. There was very limited understanding of the features within the entire product, the relationship with or impact on other features in the product. As one product information (PI) specialist put it, "some requirements were only documented in the computers of former employees, for example, leaving those involved in implementing a feature to puzzle out new requirements for themselves. From a PI perspective, it is helpful to be involved at this early stage to get an overall view of what a feature is really there for."

Furthermore, there was an indication of a potential improvement in the ability to estimate the costs and benefits of particular requested functionality, i.e. “... allows for requirements to be fleshed out with a lot more detail than previously so that a better judgment can be made about the potential benefits and costs of a feature before committing to it. This can only happen if engineers are involved, so hopefully now we will be able to avoid committing to costly but unproductive features" and "[in the past] requirements were always stated extremely briefly and left a lot to the developers imagination. Often, we would get surprises later when the scope of a requirement was realized." Other aspects mentioned by participants include the perceived benefit in using the statement template in "providing consistency in the format and writing of requirements", and "formalizing the method of capturing requirements ... should help to focus on uncovering hidden requirements". The use of the "context diagram" in developing software requirements was also found beneficial in enabling "traceability by having measurable requirements rather than one big requirement". The context diagram enabled the analysis of a new feature in the existing operational context, facilitating the identification of both functional and non-functional requirements. These aspects were perceived as short and long-term benefits of the improved practice in analyzing requirements and we

\footnotetext{
${ }^{2}$ Anecdotal data is included in quotations
} 
wanted to get further feedback in this direction. Questions addressing these aspects were included in the follow-up questionnaire, findings of which are discussed in the next section.

Twenty-three features were analyzed during forty requirements capture sessions over a six-month period (Sept/2001 - Feb/2002). During this period, the features together with the derived software requirements and traceability links were maintained in Requisite Pro database and documented in the Requirements Specification (RS). Following internal reviews at ACUS, frequent feedback on the understanding of features and the associated software requirements was sought from BI in US, to improve the validation of requirements. By including the results of the detailed requirements analysis in this document, the RS became an important vehicle for knowledge dissemination, and requirements prioritization in the interaction between ACUS Management and BI in US. The decision-makers were able to consult a document that contained not only detailed information about the required functionality, but also traceability links between high-level marketing and business requirements and software requirements. The benefits of this early detailed analysis suggest an enhanced ability to make informed decisions in release planning and managing the scope of the project. This indicated a promising improvement in the RE practice at ACUS and a follow-up questionnaire was administered at the end of the requirements analysis stage (February 2002, see plan in Figure 2) to thirty-three participants involved in a series of meetings. The findings are presented next.

\subsection{Feedback at the end of the requirements analysis stage}

The goal of the second questionnaire was twofold: (1) to elicit further feedback on the questions included in the first questionnaire as well as investigate any shift in opinions, and (2) to address issues such as participation and satisfaction with the process, immediate and long-term perceived benefits of the group session approaches to requirements analysis and the need for further training. While our intention was to collect additional feedback on improvement as recognized at the end of the 'requirements phase', in relation to the critical success factors outlined in Section 4.2, the findings reveal strong results beyond the meeting of most of these factors. We present them in the following sections in the form of themes, indicating the extent to each these factors were addressed.

\subsubsection{Effective requirements analysis sessions}

Feedback on the effectiveness of the requirements capture sessions shows sustained enthusiasm with the new practice in analyzing requirements at ACUS. As illustrated in Figure 5, there was an increase in the percentage of responses that agreed that these analysis sessions were effective in achieving a better understanding of requirements, although there was a decrease in the percentage of those who believed that they were very effective.

This result indicates that the shift in participants' opinion with respect to the effectiveness of these meetings was mainly in the positive direction. Furthermore, interview data indicates that one possible explanation for factors that lowered the effectiveness of these meetings is the perceived need for these 
meetings to involve representatives of other stakeholders with appropriate knowledge of requirements such as the Business Initiative group and the actual system end-users.

Another explanation for the skepticism about the effectiveness of these meetings may be the perception that these meetings are too slow and time-consuming in analyzing requirements. Resistance to change is one of the difficulties in managing software process improvement (Moitra, 1998). At ACUS we found a perception of increased overhead and increased demand on the developers' time. This is somewhat a natural reaction from software engineers not exposed to formal requirements analysis methods before, and who would be accustomed to a less time-consuming but less thorough analysis of the required functionality. The revised process required a cultural shift in the mind set of the engineers to move from a silo and solo based requirements analysis model to a cross functionaland team based model. The somehow natural resistance came as a reaction to most decisions being taken now in a model based on group analysis meetings rather than individual understanding and preference.

\subsubsection{More clearly defined and documented, and be tter understood requirements}

The second questionnaire included items that addressed the effectiveness of the new analysis method consisting of the use of "context diagram" and the sentence template in writing requirements, and the results are shown in Figure 6. The graph plots the percentages of responses that provided positive feedback on these items. It provides evidence in our assessment in relation to the first and second critical success factors as outlined in Section 4.2. It can be observed that overall, there was an increase in the percentage of responses, indicating that the new method contributed to reducing ambiguity in requirements and to increased understanding of requirements. A major shift is observed in the opinions that the method contributes to discovering unstated requirements ( $(\mathrm{g})$ in Figure 6$)$, from $20 \%$ at the beginning, to $60 \%$ at the end of the analysis stage. This is significant and meaningful for ACUS development team because previous RE practice did not enable developers to fully understand the impact of implementing the required functionality. This led, in the past, to making uninformed and inaccurate estimates and thus committing to functionality that in fact required much more effort than initially understood. The sustained positive response with respect to the usefulness of the context diagram (23 out of 24 responses responded positively in the second questionnaire) leads us to believe that the group analysis sessions and the supporting methods (e.g. context diagram) had significant effects in improving the requirements practice.

Similarly, a shift is observed in the responses on the benefits of the requirements sessions in improving the structure of requirements ((h) in Figure 6). The decrease in the percentage of responses, from $66 \%$ to $44 \%$, indicates that the initial enthusiasm was not shared after the involvement in a series of analysis meetings. This result is somewhat surprising since we intuitively expected that initially as participants start using the sentence template they might not fully appreciate the implicit structure that the sentence template imposes on the statements of requirements. Moreover, as participants in these sessions 
increased their familiarity with the sentence template and become accustomed to writing requirements in this way, they should in fact realize the improvement in the uniformity and structure of statements of requirements.

A related issue that is noteworthy here is the extent to which using a predefined sentence template may inhibit or constrain participants thinking about requirements. We were interested to investigate this issue for a couple of reasons: although previous research has shown that documents written in a controlled language are more comprehensible than documents written in an unrestric ted language (Shubert et al., 1995), it is also known that controlled language might be so restricted that it becomes irritating to use (Osborn and MacNish, 1996). Proponents of simplified English (and other controlled languages) have claimed that using a restricted English standard makes documents easier to read and understand but there is no convincing empirical evidence to suggest that indeed this is also the case for writing and articulating the requirements document. Hence in both questionnaires we asked the respondents to say if they think using the sentence template (see Box 1 and excel form in Figure 3), inhibited or constrained their thinking about requirements.

We expected that when participants are exposed to using a restricted sentence template initially there will be negative opinions expressed about the way in which this might inhibit or constrain their thinking and articulation of requirements. As they become more familiar with the structure, we intuitively expected that negative opinion of participants would shift to positive opinion. As seen in Figure 6 (data on (j) and (k)), the majority of responses indicate that participants did not find the sentence template inhibiting nor constraining their thinking, in either of the two questionnaires. An analysis of results using the McNemar test for the significance of changes (Siegel, 1956) $(\mathrm{N}=24, \mathrm{p}<0.001)$ finds that there was no change in the positive responses, indicating that participants did not find the template inhibiting nor constraining their thinking. This may be attributed to effective facilitation in the sessions and pre-training. Also, there are many ways one can restrict a language: a) only allow a certain set of words in the lexicon, b) only allow a certain set of syntactic constructs, c) only allow a certain semantic theory to be used to interpret the sentence, d) a combination of $a, b$, or $c$ and so on. The restrictions placed on the language in this sentence template do not seem to be of the serious type since the lexicon is unrestricted, the semantic theory is that of English language and it is only the syntax that is restricted. Hence, we may speculate that in this case restricting the grammar might not be quite as inhibiting or constraining as restricting the lexicon or the semantics of the language.

\subsubsection{Improved project scope management and requirements negotiation}

Enhanced ability to make informed decisions in release planning helped ACUS managers to better handle feature creep in this project. Tangible and immediate benefits of the revised RE process could be observed during negotiations between ACUS Management and BI, when several groups of functionality (high priority features initially) ceased to be considered after an understanding was reached that the 
customers would not be able to use this functionality effectively or that resource availability was an issue. This finding directly relates to the third critical success factor in our assessment (see Section 4.2). Namely, it suggests that the more detailed analysis of features led to an enhanced understanding of the alignment between the market needs and the product strategic direction.

The feedback from the first questionnaire also allowed us to include questions to confirm additional aspects, such as perceived increased involvement in the features being analyzed, and immediate and longterm benefits of these meetings. They are discussed next.

\subsubsection{Increased participation in the development of requirements}

Figure $\quad 7$ indicates $\quad(32$ responses in the second questionnaire) that $80 \%$ of respondents agreed (62\%: agree, $18 \%$ : strongly agree) that the participation in the analysis sessions provided them with an increased level of participation in the features for which they responsible or contributing to in

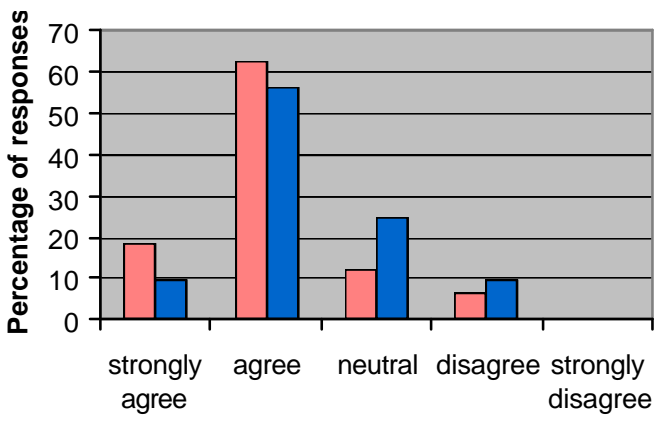

Figure 7. Agreement with increased level of involvement/sense of ownership in the features being developed

the product. A similar, although not as strong result was found in relation to perceived increased sense of ownership of the features analyzed. This is an important finding because developers felt that in the past they could have contributed more to the development of requirements. These sessions allowed them to share their knowledge and experience with other developers across system platforms, and contribute to a better understanding of required functionality. Furthermore, a significant correlation between responses on the effectiveness of requirements analysis and both the level of involvement and sense of ownership was found $(r=.75, \mathrm{p}<.01$ and $r=.58, \mathrm{p}<.01$ respectively). This finding provides additional evidence that involvement and active participation in the analysis of requirements were important to developers at ACUS and were factors associated to perceived effectiveness of the new RE practice. 


\subsubsection{Immediate benefits of improved RE practice}

Qualitative data collected in the first questionnaire suggested several immediate benefits of the improved RE practice at ACUS and more feedback was elicited in question 9 in the second questionnaire. An estimate of the internal consistency of the scale yielded a coefficient alpha of .661 , which is a relatively high rating of item homogeneity and an indication that participants responded consistently across items. Examining the results shown in Figure 8 (data from 33 responses), it can be observed that the majority of responses are in the range 60-80\%, indicating fairly positive results. More significantly, group sessions contribute to better knowledge sharing and improved communication between developers (74\% and $76 \%$ respectively). The responses on the ability to better estimate development effort for features, our fourth critical success factor in the assessment, were inconclusive. The results are mixed, i.e. $42 \%$ of respondents agreed, while other $42 \%$ were neutral in the responses. This evidence is not conclusive and it suggests that it may be too early to assess the improvement with respect to the ability to estimate effort. Our future research into the long-term effects of the improved requirements practice will report on potential improvements in estimation ability.

Similarly, a less than positive result was obtained on the ability to facilitate communication with the Business Initiative and marketing groups. A possible explanation is the perceived need to involve the participation of representatives of these groups in the requirements analysis sessions at ACUS, in order to achieve a full understanding of the rationale for particular functionality.

\subsubsection{Perceived long-term be nefits of the improved RE practice}

Feedback was collected on long-term benefits of these sessions in question 10, and results are shown in Figure 9. An estimate of the internal consistency of the scale yielded a coefficient alpha of .71, which is a relatively high rating of item homogeneity and an indication that participants responded consistently across items. While there was fairly strong agreement with benefits during design ( $72 \%$ agreed), testing

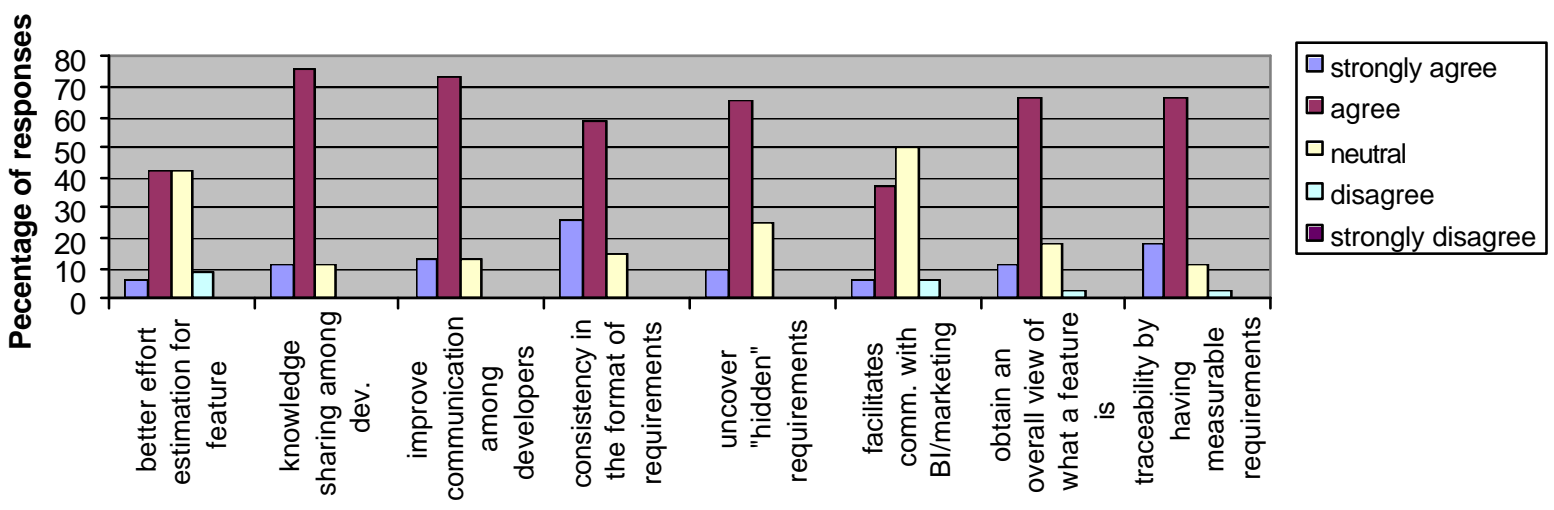

Perceived immediate benefits of requirements analysis sessions

Figure 8. Agreement with immediate benefits of the requirements analysis sessions 
and documentation (66\% agreed), the responses on the benefit during maintenance was more neutral. A possible explanation is that no participants from the support and maintenance group were involved in these sessions; hence these benefits could not be fully understood.

Furthermore, a significant correlation between the responses on the effectiveness of meetings and perceived long-term benefit during design $(r=.55, \mathrm{p}<.01)$ was found, an association that suggests that the respondents believe that effective requirements analysis has a direct relationship to improved design activities.

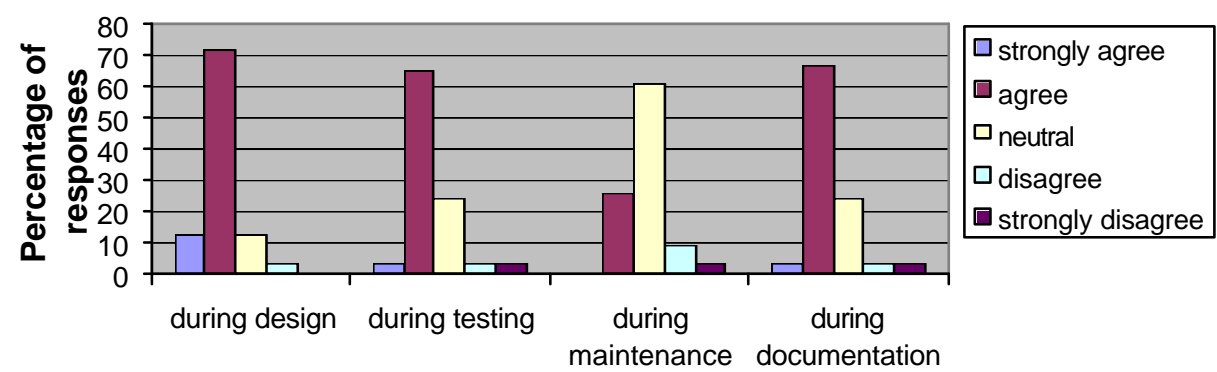

Perceived long-term benefits

Figure 9. Agreement with perceived long-term benefit of requirements analysis sessions

\subsubsection{Training}

As discussed in section 4.3.3, managing the human dimension in process improvement effort was of priority at ACUS. Training on the new methods and tools was conducted at the beginning of the requirements analysis stage. Feedback with respect to the need for more training in the requirements analysis is shown in Figure 10. The findings are somewhat mixed: among those that received training, a larger percentage of respondents felt that more training is needed; while a large percentage of respondents among those that did not receive training thought that training (hands on experience during the actual analysis sessions in their case) was sufficient.

\section{Discussion}

In this paper we reported on efforts for process improvement at one of the Unisys engineering labs, ACUS. Requirements Management was identified as one of the Key Process Area for improvement in the CMM framework and we described the initiative to address the existing challenges faced by the organization in requirements management. Our study provides evidence of process improvement in the early stages of the development and of immediate benefits of the revised RE practice.

It is important to note that the assessment did not investigate a new RE method, tool or technique. One strength of the revised RE practice at ACUS is the use of group session approach to the analysis of requirements, a proven RE technique (Macaulay, 1996). Its use to tackle the existing challenges at ACUS 
was innovative and our interest was in evaluating and reporting any improvement. Our findings suggest that the more thorough analysis of the system features in the context of the existing architecture, had positive tangible benefits. In relation to the critical success factors defined in Section 4.2, the first four factors were successfully addressed: the improved RE practice resulted in more clearly defined, documented requirements, better understood requirements, and an enhanced ability to address the market need and product strategy. Better communication, collaboration and participation of other stakeholder groups, i.e. BI and customers is, however, necessary for further improvement.

Additional findings indicate a significant association between the level of involvement in requirements analysis and perceived effectiveness of the new RE practice at ACUS, since it enabled an understanding of the features within the entire system. A similar association was found in relation to a long-term perceived benefit, in that the participants believed that effective requirements analysis has a direct relationship to improved design activities.

\subsection{Success factors in the organizational change management at ACUS}

Any process improvement involves organizational change and the evidence collected in this study indic ate that success factors at ACUS include:

1. Management commitment and support. Not only middle management, i.e. Project Manager, was actively involved in revising the process and coordinate the efforts to improve the RE practice, but the change had full support from the senior management. More importantly the project manager had a good understanding of the new process and had extensive positive experience with the proposed process in a previous role before joining ACUS. Hence it was possible for the project manager to implement the changes with confidence that it would produce the desired results. He maintained a positive attitude towards the changes throughout the project which, combined with sustained commitment to the success of the project, enabled him to coach the project team in surpassing the challenges raised during the initial resistance to cultural change.

2. Management of the human dimension of the process improvement effort. Special attention was paid to involving participants from all functional areas contributing to or affected by the project. The Project Manager played an active role in disseminating the information and providing enhanced understanding of the new process and supporting methods and tools through extensive workshops. Involvement of software engineers, test engineers and PI specialists was critical due to their first-hand knowledge of strengths and weaknesses of current process. Explaining how their involvement could contribute to the improvement initiative motivated their buy-in and adoption.

As outlined earlier the previous approach to requirements analysis was silo and solo based. This impacted the outcome of good understanding of the requirements and hence the architecture and design. For instance implementation of product-wide features varied widely across platforms. The cross functional and team based approach provided opportunities to address these problems. 
3. Tailoring the improvement initiative. The revised RE process was adapted not only to suit size and complexity of the project but also the specific technical, organizational strengths and weaknesses. ACUS had strong technical skills, but communication with the Marketing department in the business unit was weak. Thus, in the revised RE process, ACUS leveraged its engineers' skill to analyze features internally, then eliciting feedback from the marketing department.

\section{Limitations}

Although limited quantitative historical data was available from past projects, comparisons to previous projects at ACUS were still possible and indicate a significant improvement in the project. We had to rely on the input of project participants and knowledge of past practice at ACUS. This input emerged as a valuable resource since the majority of these people have been employed at ACUS for the last 5-15 years, bringing a wealth of anecdotal information about weaknesses of the software process.

Furthermore, the RE process improvements at ACUS occurred in parallel with initiatives for improvement in other process areas such as software quality assurance, project planning and project tracking. We recognize that it is very difficult to ascertain to what extent those other improvements have confounded the effects we observed in this study. Or, for that matter, to evaluate the interaction between the requirements engineering process and other processes, in particular to assess whether the RE process was of help or of hindrance to these processes and vice-versa. One could consider this to be the strongest limitation of our study and in general of assessing improvements in RE practice in an organization that is involved in a larger improvement initiative. Stronger evidence could have been brought by an assessment that designed an experiment that compared similar projects where only RE process differed, however this is a difficult ideal to achieve in an industrial, empirical investigation when organizations are motivated to improve their productivity rather than conduct scientific experimentation. In lieu of this, we are left to rely on the expert opinion of practitioners within the organization to identify individual effects of RE process changes. Although the potential for unpredictable process interaction exists even within more stable, wellestablished environments, we acknowledge that this interaction requires further study and it is the subject of our future research.

\section{Conclusions}

Further empirical assessments at ACUS are necessary to fully understand the improvement that the revised RE practice brought about. Two of our critical success factors, improvement in requirements change management and enhanced estimation ability, could not be addressed by the evidence in this early assessment. Therefore, studying the evidence on these factors represents the topic of our future research. We recognize that assessing long-term effects of the RE improvement initiative at ACUS needs careful 
attention to interaction and the resulting integrated effects of several process improvement areas. Improvements in the requirements practice will very likely be influenced or influence improvements in areas such as project planning and software quality assurance. That is where this paper contributes to evidence on immediate benefits of improved RE practice in early stages of the project, before interaction with other process areas could be significant.

At the time of submission of this paper to the Journal of Empirical Software Engineering (one year after the study was completed), more project information became available that is noteworthy. While not directly a result of our study, it provides insights into effects of process improvement. First, ACUS has since attained CMM Level 2 certification and the score for the KPA Requirements Management had improved from 5.4 to 7.4. This suggests that the revised RE practice also contributed to software process improvement in the context of CMM assessment. While CMM identifies that there was a good process in place and it was practiced, our assessment provides evidence into the effectiveness of the revised RE process and hence its role in software process improvement. Secondly, customer satisfaction surveys and number of defects showed a marked improvement in the overall result of the project. While improvements in all Level 2 assessment areas contributed to the end result, customer satisfaction is positive ly correlated to fulfillment of customer feature requests, and thus can be utilized as a good indicator of tangible improvement in the RE process.

In conclusion, we believe that findings of our study bring a significant contribution to the evidence on software process and in particular Requirements Engineering process improvement areas. Given the scarcity of RE-related SPI initiatives in the literature, these findings should not only be of interest to researchers in Requirements Engineering who seek to empirically assess methods, processes and techniques, or develop theories of RE improvement; we hope they are also of special interest to practitioners who are seeking ripe fruits (ICRE, 1996) of RE research that can be applied within projects almost immediately with direct and tangible benefits.

\section{Acknowledgements}

Thanks are due to study participants at ACUS. This research was funded by the Natural Sciences and Engineering Research Council of Canada and a post-doctoral fellowship at University of Technology, Sydney, Australia. 


\section{References}

1. Basili, V., McGarry F., Pajerski R., and Zelkowitz M., Lessons Learned from 25 Years of Process Improvement: The Rise and Fall of the NASA Software Engineering Laboratory, Proceedings of the 24th International Conference on Software Engineering (ICSE) 2002, Orlando, Florida, May 2002.

2. Brodman J, and Johnson D., Return on Investment from software process improvement as measured by U.S. Industry, In Crosstalk, 9(4): 23-29, April 1996

3. Capability Maturity Model for Software, CMU/SEI-91-TR-24, Software Engineering Institute, Carnegie Mellon University

4. Chaos Report, Few IS Project Come in on Time, on Budget, Computer World 12, Dec., 20, 1995

5. Dion, R. Process Improvement and the Corporate Balance Sheet, IEEE Software, 10(4), July/Aug., 1993, 28-35

6. Diaz, M. and Sligo, J. How Software Process Improvement Helped Motorola, IEEE Software, 14(5), Sept/Oct. 1997, 75-81

7. El Emam K., Birk A., Validating the ISO/IEC 15504 measure of software requirements analysis process capability. IEEE Transactions on Software Engineering 26(6) 2000, pp 541-566.

8. El Emam K, and Briand L., Costs and Benefits of Software Process Improvement, International Software Engineering Research Network Technical Report ISERN-97-12, 1997.

9. El Emam, K., Drouin, J-N. and Melo, W. (eds.): SPICE: The Theory and Practice of Software Process Improvement and Capability Determination. IEEE CS Press, 1998.

10. Hall, T., Beecham S., and Rainer A., Requirements problems in twelve software companies: an empirical analysis, IEE proceedings Software, August 2002.

11. Halligan, R. TAA's SE Training Courseware, Halligan Corporation Pty Ltd, 2000

12. Herbsleb JD, Goldenson DR, A systematic survey of CMM experience and results, In proceedings of the $18^{\text {th }}$ International conference on Software Engineering, Berlin, Germany, 1996, pp 323-330

13. Humphrey, W.S., Snyder, T.R. and Willis, R.R. Software Process Improvement at Hughes Aircraft, IEEE Software, 8(4), July/Aug. 1991, 11-23

14. ISO/IEC 15504, Information technology - Software process assessment, Technical report Type 2, 1998.

15. Johnson, A., Software Process Improvement experience in the DP/MIS function, In proceedings of the IEEE International Conference on Software Engineering, 1994. 
16. Kotonya G., Sommerville I., Requirements Engineering Processes and Techniques, John Wiley, 1997.

17. Laporte, C.Y. and Trudel, S. Addressing the people issues of process improvement activities at Oerlikon Aerospace, Software Process, Improvement and Practice, 4(4), 187-198,1998

18. Lauesen, S. and Vinters, O. Preventing Requirements Defects, Requirements Engineering Journal 6 (1), 37-50, 2001

19. Macaulay, L. Requirements Engineering, Springer-Verlag, 1996

20. Moitra, D. Managing change for software process improvement initiatives: a practical experiencebased approach, Software Process, Improvement and Practice, 4(4),199-208,1998

21. Osborne, M. and MacNish, C. Processing Natural Language Software Requirements Specification, Proceedings of $2^{\text {nd }}$ IEEE International Conference on Requirements Engineering, ICRE96, Colorado Springs, Colorado, 229-236, 1996

22. Paulk M., Curtis B., Chrissis M. and Weber C., Capability Maturity Model for software, Version 1.1, CMU/SEI-93-TR-24, Software Engineering Institute USA, 1993.

23. Quality standards: Quality management and quality assurance standards, Int. Org. for Standardization, 1987

24. ICRE1996: Ripe Fruit in RE: Techniques you can exploit NOW, Panel at the $2^{\text {nd }}$ International Conference of Requirements Engineering, p. 42, 1996

25. Sawyer, P., Sommerville, I. and Viller, S. Improving the Requirements Process, Proceedings of the $4^{\text {th }}$ International Workshop on Requirements Engineering: Foundation of Software Quality (REFSQ'98), 71-84, Pisa, Italy, July 1998.

26. Shubert, S., Spyridakis, J., Holmback, H. and Coney, M. B. The Comprehensibility of simplified English in procedures, Journal of Technical Writing and Commune, 25(4), 347-369, 1995

27. Siegel, S. Nonparametric Statistics, McGraw-Hill, 1956

28. SEI, 1995: Software Engineering Institute: The Capability Maturity Model: Guidelines for Improving the Software Process. Addison Wesley, 1995.

29. Sommerville, I., Private Communications, 2003.

30. Sommerville, I. and Sawyer, P., Requirements Engineering, A Good Practice Guide, Wiley, Great Britain, 1997.

31. Stelzer, D. and Mellis, W. Success factors of organizational change in software process improvement, Software Process, Improvement and Practice, 4(4), 227-250,1998

32. Wohlwend H, Rosenbaum S., Software Process improvement in an international company, In proceedings of the International Conference on Software Engineering, pp 212-220 1993. 


\section{APPENDIX}

\section{Questionnaire}

$1^{*}$. The intended objectives of these sessions are to analyze the high-level requirements, to capture and develop low-level requirements, and increase shared understanding of these requirements. Please rate the effectiveness of these sessions with respect to achieving these objectives:

$\begin{array}{llcl} & \text { Somewhat not } \\ \text { Very effective } & \text { Effective } & \text { Neutral } & \text { effective }\end{array}$

$2 *$ In which of the following did you find the Excel form (i.e. Parser) to break down the requirement helpful?

$\square \quad$ Reducing ambiguity in requirements

$\square \quad$ Reducing the time spent in capturing the requirements

$\square \quad$ Capturing correct requirements

$\square \quad$ Capturing complete requirements

$\square \quad$ Capturing consistent requirements

$\square \quad$ Increasing the shared understanding of the requirements

$\square \quad$ Discovering new (or unstated) requirements

$\square \quad$ Improving the structure of requirements

$\square \quad$ Discovering the relationships between individual requirements

$\square \quad$ Other (please specify)

$3^{*}$. Did you find that the use of the Excel form (i.e. sentence template) to break down the requirement:

Inhibited or

$\square \quad$ Constrained your thinking about or expressing the requirements in any way?

If yes, please explain how?

4*. Did you find the context diagram helpful in analyzing the requirements?

Yes

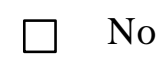

\footnotetext{
* Question appeared in both questionnaires
} 


\section{Please explain why?}

$5^{*}$. Do you feel that you now have sufficient training and knowledge for using this process again?

$$
\begin{aligned}
& \square \quad \text { Yes, suffic ient training } \\
& \square \quad \text { No, more training is needed }
\end{aligned}
$$

$6^{+}$. Do you believe that these sessions will bring about any improvement over previous requirements analysis practices at ACUS? Please explain why and how.

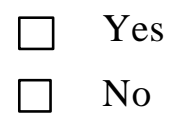

7. Do you feel that by participating in these sessions you gained an increased level of involvement in the features for which you are responsible/contributing to for this product?

Strongly agree $\quad$ Agree $\quad$ Neutral Disagree $\quad$ Strongly disagree

8. Do you feel that by participating in these sessions you gained an increased sense of ownership of the features for which you are responsible/contributing to for EAE 3.3?

Strongly agree $\quad$ Agree $\quad$ Neutral $\quad$ Disagree $\quad$ Strongly disagree

\footnotetext{
${ }^{+}$Question appeared only in the first questionnaires
} 
9. Do you agree that the following represent an immediate benefit of these sessions?

\begin{tabular}{|c|c|c|c|c|}
\hline $\begin{array}{c}\text { Strongly } \\
\text { agree }\end{array}$ & Agree & Neutral & Disagree & $\begin{array}{l}\text { Strongly } \\
\text { disagree }\end{array}$ \\
\hline 1 & 2 & 3 & 4 & 5 \\
\hline 1 & 2 & 3 & 4 & 5 \\
\hline 1 & 2 & 3 & 4 & 5 \\
\hline 1 & 2 & 3 & 4 & 5 \\
\hline 1 & 2 & 3 & 4 & 5 \\
\hline 1 & 2 & 3 & 4 & 5 \\
\hline 1 & 2 & 3 & 4 & 5 \\
\hline 1 & 2 & 3 & 4 & 5 \\
\hline 1 & 2 & 3 & 4 & 5 \\
\hline
\end{tabular}

10. Do you agree that there is any long-term benefit of these sessions?

- Helps uncover "hidden" requirements

- Facilitates further communication with $\mathrm{BI} /$ Marketing

- Get an overall view of what a feature is really there for

- Traceability by having measurable requirements rather than one big requirement

- Other (please specify)

of these sessions?

$\begin{array}{ccccc}\begin{array}{c}\text { Strongly } \\ \text { agree }\end{array} & \text { Agree } & \text { Neutral } & \text { Disagree } & \begin{array}{c}\text { Strongly } \\ \text { disagree }\end{array} \\ 1 & 2 & 3 & 4 & 5 \\ 1 & 2 & 3 & 4 & 5 \\ 1 & 2 & 3 & 4 & 5 \\ 1 & 2 & 3 & 4 & 5 \\ 1 & 2 & 3 & 4 & 5\end{array}$

